\title{
2.
}

\section{Motus corporum coelestium in medio resistente.}

(Anct. Dr. Sohncke, Regiom.)

Perturbationes elementorum orbitae corporis coelestis determinare ad difficillima astronomiae analyticae problemata adhuc pertinet. Omnia eniur huc pertinentia aut parva excentricitate posita, ita ut ejus potestates primis majores negligendae sint, aut magna excentricitate quadraturis mechavicis, quae dicuntur, hucusque sunt soluta. Specialem quaestionem huius generis, in qua excentricitatis magnitudo finibus omnino non circumscribitur, quantum fieri potest, accuratius solvere nobis proposuimus. Hoc est problema :

"Determinare variationes elementorum orbitae corporis coelestis in "medio resistente moti."

\section{\$. 1.}

Medium solem eircumdans, in quo nonnulli corpora coelestia moreri ponunt, pro fluido est habendum, euius densitas in ratione inversa est cum quadrato distantiae a sole, igitur $=\frac{m}{r^{2}}$, si $r$ radium vectorem significat et $m$ factorem constantem. Cum vero medium resistens inclinationem plani orbitae ad fixum quoddam planum atque angulum inter lineam nodorum et lineam absidarum comprehensum mutare nen posse pateat, de plano tantum loqui iam sufficit, ita ut tertia linea coordinata prorsus omittatur. Sit igitur $\partial s$ sive $\checkmark\left(\partial x^{2}+\partial y^{2}\right)$ elementum viae' ${ }^{\prime}$ quod a corpore in momento $\partial t$ percurritur, resistentia, qua directio viae mutatur, ipsi $\frac{m}{r^{2}} \cdot\left(\frac{\partial s}{\partial t}\right)^{2}$ erit aequalis, dummodo, ut plerumque, inter resistentiam et quadratum celeritatis corporis moti rationem directam esse statuamus. Haec: resistentia secundum direetiones linearum coordinatarum rectum angulum formantium $x$ et $y$ decomposita praebet:

$$
\text { 2f. } A=\frac{m}{r^{2}} \cdot \frac{\partial x \cdot \partial s}{\partial t^{2}} ; \quad B=\frac{m}{r^{2}} \cdot \frac{\partial y \cdot \partial s}{\partial t^{2}},
$$

si resistentiam in directione axis $x$ per $A$, et in directione axis $y$ per $B$ notamus. 
2. Sohncke, motus corporum coelestium in medio resistente.

In sequentibus significalit:

$r$ : radium vectorem,

$\alpha$ : semiaxem majorem,

$e$ : rationem excentricitatis ad semiaxem majoren,

$h h=p:$ semiparametrum,

$w$ : angulum inter axem majorem orbitae et axem coordinatarum $x$,

$v$ : angulum inter axem coordinatarum $x$ et radium vectorem, ita ut $(v-w)$ veram anomaliam exprimat,

$T$ : tempus perihelii.

Si corpus coeleste circum solem movetur, nulla alia vi sollicitatum nisi attractiva solis, aequationes omnibus notae exstant hae:

$$
\text { B. } \quad \frac{\partial^{2} x}{\partial t^{2}}+\frac{x}{r^{3}}=0 ; \quad \frac{\partial^{2} y}{\partial t^{2}}+\frac{y}{r^{2}}=0
$$

ubi $x$ et $y$ coordinatae sunt, quarum initium in sole.

Lx his constat deduci posse sequentes:

$$
\text { c. } \begin{cases}\text { 1. } & \frac{\partial x^{2}+\partial y^{2}}{\partial t^{2}}=\frac{2}{r}-\frac{1}{a}, \\ \text { 2. } & \frac{x \partial y-y \partial x}{\partial t}=r p \\ \text { 3. } & e \cos w=-\frac{x}{r}-y \cdot \frac{\partial y \cdot \partial x}{\partial t^{2}}+x \cdot \frac{\partial y^{2}}{\partial t^{2}} \\ \text { 4. } & e \sin w=-\frac{y}{r}-x \cdot \frac{\partial x \cdot \partial y}{\partial t^{2}}+y \cdot \frac{\partial x^{2}}{\partial t^{2}}\end{cases}
$$

atque si ponitur:

$$
x=r \cos v ; \quad y=r \sin v \text { : }
$$

$\left\{\begin{aligned} \text { 5. } r & =\frac{a(1-e e)}{1+e \cos (v-w)}=\frac{p}{1+e \cos (v-w)}, \\ \text { 6. } t+T & =a^{\frac{3}{2}} \operatorname{arc}\left\{\cos =\frac{e+\cos (v-w)}{1+e \cos (v-w)}\right\}-a^{\frac{3}{2}} \cdot e r(1-e e) \cdot \frac{\sin (v-w)}{1+e \cos (v-w)}, \\ \text { 7. } \frac{\partial r}{\partial t} & =\frac{e}{\sqrt{p}} \cdot \sin (v-w), \\ \text { 8. } \frac{\partial v}{\partial t} & =\frac{V_{p}}{r r} .\end{aligned}\right.$

Si vero alia vis praeter vim solis corpus sollicitat, in utraque aequatione ( $\mathfrak{B}$.) est terminus addendus, qui ab illa secundum coordinatas decomposita vi pendet, ita ut habeamus:

$$
\text { D. } \frac{\partial^{2} x}{\partial t^{2}}+\frac{x}{r^{3}}+A=0, \frac{\partial^{2} y}{\partial t^{2}}+\frac{y}{r^{3}}+B=0,
$$

ubi $A$ et $B$ valores in (A.) memoratos habent. 
Ut hae integrentur, praeclara illa theoria variationis quantitatum con-

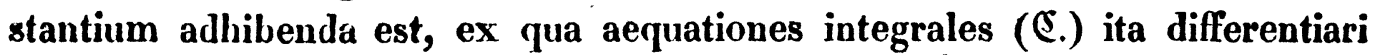
debent, ut constantes et $\frac{\partial x}{\partial t}$ et $\frac{\partial y}{\partial t}$ tanquam variabiles existimentur, $x, y$, $t$ vero tanquam constantes, tum pro $\frac{\partial^{2} x}{\partial t^{2}}$ et $\frac{\partial^{2} y}{\partial t^{2}}$ illi nóodo termini ex aequationibus (D.) ponantur, qui a viribus perturbantibus pendent, ita ut fit:

quo pacto eruuntur:

$$
\frac{\partial^{2} x}{\partial t^{2}}=-A ; \quad \frac{\partial^{2} y}{\partial t^{2}}=-B ;
$$

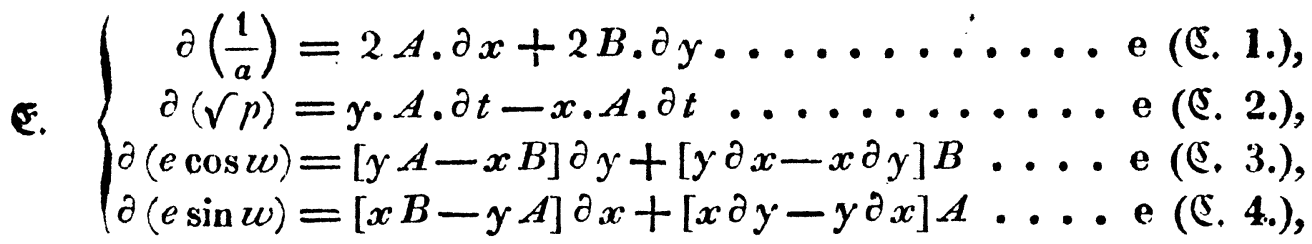

hinc prodeunt valores variationum $\dot{d} e$ et $\partial w$ ope formularum:

$$
\begin{aligned}
& \partial e=\cos w \cdot \partial(e \cos w)+\sin w \partial(e \sin w), \\
& \partial w=\frac{1}{e} \cos w \cdot \partial(e \sin w)-\frac{1}{e} \sin w \partial(e \cos w) .
\end{aligned}
$$

Porro fit :

$$
\text { ๔. } \begin{aligned}
\partial T= & -\frac{3}{2} a^{\frac{b}{2}} \partial\left(\frac{1}{a}\right) \cdot\left[\operatorname{arc}\left(\cos =\frac{e+\cos (v-w)}{1+e \cos (v-w)}\right)-\frac{e V(1-e e) \cdot \sin (v-w)}{1+e \cos (v-w)}\right] \\
& -a^{\frac{2}{2}} V(1-e e) \cdot\left[\frac{(1-e e) \partial w}{[1+e \cos (v-w)]^{3}}+\frac{[2+e \cos (v-w)] \sin (v-w) \partial e}{[1+e \cos (v-w)]^{2}}\right] \ldots \text { e (C. 6.) }
\end{aligned}
$$

Ut hi valores formam ad computandum aptissimam induant et tempus $t$ et anomaliam veram $(v-w)$ anomalia excentrica $u$ exprimi convenit, quod ope formulae:

$$
\operatorname{tang} \frac{x}{2}(v-w)=V\left(\frac{1+e}{1-e}\right) \operatorname{tang} \frac{x}{2} u
$$

Reri potest, unde oriuntur:

$$
\begin{aligned}
\sin (v-w) & =r(1-e e) \frac{\sin u}{1-e \cos u} ; \quad \cos (v-w)=\frac{\cos e-u}{1-e \cos u} \\
r & =a(1-e \cos a) ; \quad t+T=a^{\frac{1}{2}}[u-e \sin u] ;
\end{aligned}
$$

$\partial t=a^{\frac{3}{2}}[1-e \cos u] \partial u ; \quad \partial r=a e \cdot \sin u . \partial u ; \quad \partial v=r(1-e e) \cdot \frac{\partial u}{1-e \cos u} ;$

$$
\begin{aligned}
x & =a \cdot[(e+\cos u) \cdot \cos w-V(1-e e) \cdot \sin u \cdot \sin w], \\
y & =a \cdot[(e+\cos u) \cdot \sin w+V(1-e e) \cdot \sin u \cdot \cos w], \\
\partial x & =-a \cdot[V(1-e e) \cdot \cos u \cdot \sin w+\sin u \cdot \cos w] \cdot \partial u, \\
\partial y & =a \cdot[V(1-e e) \cdot \cos u \cdot \cos w-\sin u \cdot \sin w] \cdot \partial u, \\
\partial s & =a \cdot r\left(1-e e \cos u^{2}\right) \cdot \partial u_{2}
\end{aligned}
$$

Creutes Journal a M. Bd. X. Ha. 1. 


$$
\begin{aligned}
& A=-\frac{m}{a^{3}} \cdot \frac{[V(1-e e) \cos u \cdot \sin w+\sin u \cdot \cos w] \cdot[1+e \cos u]}{[1-e \cos u]^{3} V\left(1-e e \cos u^{2}\right.}, \\
& B=\frac{m}{a^{3}} \cdot \frac{[V(1-e e) \cos u \cdot \cos w-\sin u \cdot \sin w] \cdot[1+e \cos u]}{[1-e \cos u]^{3} V\left(1-e e \cos u^{2}\right)}
\end{aligned}
$$

\section{Unde aequationes ( $\left.E_{0}\right)$, commutantur in has:}

$$
\begin{aligned}
& \text { (I. } \partial\left(\frac{1}{a}\right)=2 m \cdot \frac{(1-e e)^{2}}{p p} \cdot\left[\frac{1+e \cos u}{1-e \cos u}\right]^{2} \frac{\partial u}{\sqrt{\left(1-e e \cos u^{2}\right)^{2}}} \\
& \text { II. } \partial(\checkmark p)=-m \cdot \frac{1-e e}{\sqrt{p}} \cdot\left[\frac{1+e \cos u}{1-e \cos u}\right] \frac{\partial}{\sqrt{\left(1-e e \cos u^{2}\right)}} \text {, } \\
& \text { F. }\left\{\text { III. } \partial w=-2 m \cdot \frac{(1-e e)^{\frac{3}{2}}}{p e} \cdot \frac{[1+e \cos u] \cdot \sin u}{[1-e \cos u]^{2}} \cdot \frac{\partial u}{\sqrt{ }\left(1-e e \cos u^{2}\right)}\right. \text {, } \\
& \text { [V. } \partial T=m \cdot \frac{V p}{V(1-e e)} \cdot\left[\frac{2(1+e e)}{e}+e(1+e \cos u)\right] \cdot \frac{[1+e \cos u] \cdot \sin u \cdot \partial u}{[1-e \cos u]^{2} V\left(1-e e \cos u^{2}\right)} \\
& -3 m \cdot \frac{V_{p}}{V(1-e e)} \cdot\left[\frac{1+e \cos u}{1-e \cos u}\right]^{2} \cdot \frac{u \partial u}{r\left(1-e e \cos u^{2}\right)} \text {. }
\end{aligned}
$$

Variatio $\partial e$ hie non est nominata, quia ex aequatione

statim sequitur:

$$
p=a(1-e e)
$$

$$
\partial e=-\frac{p}{2 e} \cdot \partial\left(\frac{1}{a}\right)-\frac{1-e e}{e \gamma p} \cdot \partial(r p) .
$$

\section{§. 2.}

Tertia tantum aequatio integratione algebraica gaudet. Posito enim :

formula :

$$
e \cdot \cos u=\psi \text {. }
$$

$$
w=-2 m \cdot \frac{(1-e e)^{\frac{2}{2}}}{p e} \cdot \int_{0}^{u} \frac{[1+e \cos u] \cdot \sin u}{[1-e \cdot \cos u]^{2}} \cdot \frac{\partial u}{\sqrt{\left(1-e e \cos u^{2}\right)}}
$$

transit in sequentem:

igitur fit:

$$
w=-2 m \cdot \frac{(1-e e)^{\frac{3}{2}}}{p^{e \cdot e}} \int \frac{\operatorname{cotang}^{2} \frac{x}{2} \psi \cdot \partial \frac{1}{2} \psi}{\sin ^{2} \frac{1}{2} \psi}\left\{\begin{array}{l}
\text { a: } \psi=\operatorname{arc}(\cos =e) \\
\text { ad: } \psi=\psi \text { usque. }
\end{array}\right\},
$$

sive :

$$
\begin{aligned}
& w=\frac{2}{3} m \cdot \frac{(1-e)^{\frac{5}{2}}}{p e e} \operatorname{cotang} \frac{1}{2} \psi^{3}-\frac{2}{3} m \cdot \frac{(1+e)^{2}}{p e e}, \\
& w=\frac{2}{3} m \cdot \frac{(1+e)^{2}}{p e e} \cdot\left\{\left[V\left(\frac{1-e}{1+e}\right) \cdot \operatorname{cotang} \frac{1}{2} \psi\right]^{3}-1\right\},
\end{aligned}
$$

(III $\left.{ }^{*}\right) \quad w=\frac{2}{3} m \cdot \frac{(1-e)^{2}}{p e e} \cdot\left\{\left[V\left(\frac{1-e}{1+e}\right) /\left(\frac{1+e \cos u}{1-e \cos u}\right)\right]^{2}-1\right\}$. 


\section{§. 3.}

Prioris ambae aequationes $I$ et II. simili modo sunt tractandae, qua de causa hic non separatim de iis agamus. Cunstituamus *):

$$
\begin{gathered}
\frac{(1+e)(1-\cos u)}{2(1-e \cos u)}=\sin \varphi^{2} ; \frac{2 \sqrt{e}}{1+1}=\lambda ; \frac{1-e}{1+e}=r(1-\lambda \lambda)=\lambda^{\prime} ; \\
r\left(1-\lambda \lambda \sin ^{2} \varphi\right)=\Delta \varphi,
\end{gathered}
$$

unde accipimus :

$$
\cos u=-\frac{1+\lambda^{\prime}}{1-\lambda^{\prime}} \cdot \frac{\lambda^{\prime}-\Delta^{2} \phi}{\lambda^{\prime}+\Delta^{2} \phi} ; \quad \sin u=2\left(1+\lambda^{\prime}\right) \vee \lambda^{\prime} \cdot \frac{\sin \varphi \cdot \cos \varphi}{\lambda^{\prime}+\Delta^{2} \varphi} ;
$$

$1+e \cos u=\frac{2 \cdot \Delta^{2} \varphi}{\lambda^{\prime}+\Delta^{2} \varphi} ; 1-e \cos u=\frac{2 \lambda^{\prime}}{\lambda^{\prime}+\Delta^{2} \varphi} ; r\left(1-e e \cos u^{2}\right)=\frac{2 V\left(\lambda^{\prime}\right) \cdot \Delta \varphi}{\lambda^{\prime}+\Delta^{2} \varphi} ;$

$$
\partial u=2\left(1+\lambda^{\prime}\right) r \lambda^{\prime} \cdot \frac{\partial \varphi}{\lambda^{\prime}+\Delta^{2} \varphi} ; \quad \frac{\partial u}{r\left(1-e e \cos u^{2}\right)}=\left(1+\lambda^{\prime}\right) \cdot \frac{\partial \varphi}{\Delta \varphi} .
$$

Hi valores in aequationibus prius citatis (I. et II.) -positi, eas commutant in sequentes:

Est vero:

$$
\partial\left(\frac{1}{a}\right)=\frac{32 \cdot m}{l_{p}\left(1+\lambda^{\prime}\right)^{3}} \cdot \Delta^{3} \varphi, \partial \varphi ; \quad \partial(\sqrt{p})=-\frac{4 m}{\sqrt{p}\left(1+\lambda^{\prime}\right)} \cdot \Delta \varphi . \partial \varphi .
$$

atque

$$
\int_{0}^{\Phi} \Delta \varphi \cdot \partial \varphi=\mathbf{E}(\varphi)
$$

$$
\int_{0}^{\varphi} \Delta^{3} \varphi \cdot \partial \varphi=\frac{1}{3} \lambda \lambda \cdot \sin \varphi \cdot \cos \varphi \cdot \Delta \varphi+\frac{2}{3}\left(1+\lambda^{\prime} \lambda^{\prime}\right) \mathbf{E}(\varphi)-\frac{1}{3} \lambda^{\prime} \lambda^{\prime} \cdot \mathbf{F}(\varphi) .
$$

Itaque aequationibus $I$. et II. integratis obtinemus :

$I^{*} . \quad \frac{1}{a}=\frac{32 \cdot m}{3 p p\left(1+\lambda^{\prime}\right)^{3}} \cdot\left[\lambda \lambda \cdot \sin \varphi \cdot \cos \varphi \cdot \Delta \varphi+2\left(1+\lambda^{\prime} \lambda^{\prime}\right) \mathbf{E}(\varphi)-\lambda^{\prime} \lambda^{\prime} F(\varphi)\right]$,

$$
\text { II*. } r p=-\frac{3 m}{\sqrt{p\left(1+\lambda^{\prime}\right)}} \cdot \mathrm{E}(\varphi) \text {. }
$$

§. 4.

Ex aequationibus (F.) in prima sectione accitis una tantum integrando restat et ea quidem, cuius integratione variationem temporis perihelii adipiscimur. Illic enim habuimus :

IV. $\partial T=\frac{m \cdot V p}{V(1-e e)} \cdot\left[\frac{2(1+e e)}{e}+e(1+e \cdot \cos u)\right] \cdot \frac{[1+e \cos u] \cdot \sin u \cdot \partial u}{[1-e \cos u]^{2} V\left(1-e e \cos u^{2}\right)}$

ex (no sequitur:

$$
-3 m \cdot \frac{V_{p}}{\sqrt{(1-e e)}} \cdot\left[\frac{1+e \cdot \cos u}{1-e \cdot \cos u}\right]^{2} \frac{u}{\sqrt{\left(1-e e \cos u^{2}\right)}}
$$

$$
\text { C. } T=\frac{m V_{P}}{V\left(1-e^{\varepsilon}\right)} \cdot[P-3 Q]
$$

-) Hic et in iis, quae sequuntur, significandi genere utar, quod Cl. Legendre et $J$ a co $i$ in scriptis de functionibus, quae dicuntur ellipticae transcendentes, in usum vocarunt. 
ubi valet :

$P$ idem ac: $\int_{0}^{u}\left[\frac{2(1+e e)}{e}+e(1+e \cdot \cos u)\right] \frac{[1+e \cos u] \cdot \sin u \cdot \partial u}{[1-e \cos u]^{2} V\left(1-e e \cos u^{2}\right)}$ et

$Q$ idem ac: $\int_{0}^{u}\left[\frac{1+e \cos u}{1-e \cos u}\right]^{2} \frac{u \partial u}{\sqrt{\left(1-e e \cos u^{2}\right)}}$.

Tantum ipsum $P$ quantitatibus finitis determinari potest. Si enim eadem substitutione ac in sectione antecedente utimur, obtinemus : sive:

$$
P=\int_{0}^{\prime} \frac{2 \lambda \lambda}{\sqrt{ } \lambda^{\prime}} \cdot\left[\frac{3-2 \lambda^{\prime}+3 \lambda^{\prime} \lambda^{\prime}}{\lambda^{\prime}\left(1-\lambda^{\prime}\right)^{2}}-\frac{1}{\lambda^{\prime}+\Delta^{2} \varphi}\right] \sin \varphi \cdot \cos \varphi \cdot \Delta \varphi \cdot \partial \varphi,
$$

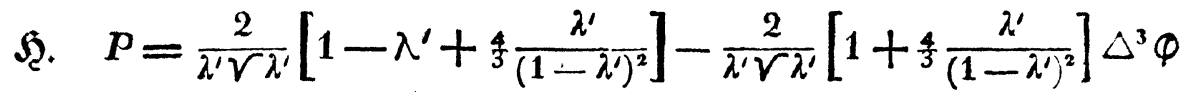

$$
+\frac{2}{\sqrt{\lambda^{\prime}}} \Delta \varphi+2 \cdot \operatorname{arc}\left[\operatorname{tang}=\frac{V \lambda^{\prime}(1-\Delta \varphi)}{\lambda^{\prime}+\Delta \varphi}\right] \text {. }
$$

Quod ad alteram partem integralis quaesiti attinet, eadem integrandi facilitate non fruimur. Primum integratio per partes, quae dicitur, instituatur, quo facto eruimus:

$$
\text { ฐ. } Q=u \cdot R-\int_{0}^{u} R \partial u,
$$

si :

per $R$ reddimus.

$$
\int_{0}^{u}\left[\frac{1+e \cos u}{1-e \cos u}\right]^{2} \frac{\partial u}{\sqrt{\left(1-e e \cos u^{2}\right)}}
$$

Ipsum $R$ jam determinatum est, cum variationem semiaxsis majoris evolvebamus. (\$. 3.), ubi prodiit:

R. $R=\frac{1+\lambda^{\prime}}{3 \lambda^{\prime} \lambda^{\prime}}\left[\lambda \lambda \cdot \sin \varphi \cdot \cos \varphi \cdot \Delta \varphi+2\left(1+\lambda^{\prime} \lambda^{\prime}\right) \mathbf{E}(\varphi)-\lambda^{\prime} \lambda^{\prime} \mathbf{E}(\varphi)\right] \cdot$ Ut $\int_{0}^{u} R . \partial u$ facillime obtineamus, $R$ in quatuor partes dividere libet, quippe aequatis,

$$
\begin{array}{ll}
U=\int \frac{\left[1+e e \cos u^{2}\right] \cdot \cos u \cdot \partial u}{\left[1-e e \cos u^{2}\right] 2 V\left(1-\cos u^{2}\right)}, & V=\int \frac{\partial u}{\sqrt{\left(1-e e \cos u^{2}\right)},} \\
V_{1}=\int \frac{\partial u}{\left[1-e e \cos u^{2}\right] V\left(1-e e \cos u^{2}\right)}, & V_{2}=\int \frac{\partial u}{\left[1-e e \cos u^{2} V\left(1-e e \cos u^{2}\right)\right.}
\end{array}
$$

6it:

$$
\int_{0}^{u} R \cdot \partial u=4 e \int_{0}^{u} U \cdot \partial u+\int_{0}^{u} V_{0} \partial u-8 \int_{0}^{u} V_{2} \cdot \partial u+8 \int_{0}^{u} V_{2} \cdot \partial u \text {. }
$$

Ad primum terminum determinandum ponamus:

$$
\frac{\sin u}{r\left(1-e e \cos u^{2}\right.}=\sin \xi ; \quad r\left(1-e e \sin \xi^{2}\right)=\Delta \xi,
$$

unde fluunt:

$\sin u=r(1-e) \cdot \frac{\sin \xi}{\Delta \xi} ; \cos u=\frac{\cos \xi}{\Delta \xi}, \partial u=r(1-e e) \cdot \frac{\partial \xi}{\Delta^{2} \xi} ; r(1-e e)=e^{\prime}$, 
quo pacto fit:

$$
U=\frac{1}{e^{\prime 4}} \cdot\left[1+e e-\frac{2}{3} e e \sin \xi^{2}\right] \cdot \sin \xi
$$

atque :

$$
\int_{0}^{u} U \partial u=\frac{4}{3 \cdot e^{12}} \cdot \sin \frac{r}{2} \xi^{2}+\frac{1+3 e e}{3 e \cdot e^{12}} \operatorname{arc}\left[\operatorname{tang}=\frac{2 e e^{\prime} \sin \frac{x}{2} \xi^{2}}{e^{\prime} e^{\prime}+\frac{c}{c e \cos \xi}}\right] .
$$

§. 5.

Hucusque omnia aut quantitatibus finitis aut functionibus ellipticis primae et secundae speciei determinari potuerunt, ad reliqua vero integralia $V, V_{3}, V_{2}$ definienda, illae praeclarae functionum ellipticarum evolutiones, quas primus $\mathrm{Cl}$. Ja cobi dedit, advocentur necesse est.

Iunc in finem ponere convenit :

atque

$$
u=\frac{\pi}{2}-w
$$

ita ut fit:

$$
w=\operatorname{coam} \frac{2 \pi x}{\pi}(\bmod . e)
$$

$$
\int_{0}^{w} \frac{\partial w}{\sqrt{\left(1-e e \sin w^{2}\right)}}=K-\frac{2 K x}{\pi},
$$

atque am $\frac{2 \pi x}{\pi}$ idem ac $\xi$ in sectione antecedente.

Si praeterea redditur:

$$
\begin{array}{ll}
V\left(1-e e \sin ^{2} a m \frac{2 K x}{\pi}\right) & \text { per } \Delta \operatorname{am} \frac{2 K x}{\pi} \text { et } \\
\int_{a}^{x} \Delta^{2} a m . \partial x & \text { per } \left.\operatorname{Eam} \frac{2 K x}{\pi}\right)
\end{array}
$$

obtinemus :

$$
\begin{aligned}
& V=\frac{2 K x}{\pi}, \\
& V_{2}=\frac{1}{e^{\prime} e^{i}} \cdot \frac{2 K}{\pi} \cdot \int_{a}^{x} \Delta^{2} \operatorname{am} \frac{2 K x}{\pi} \partial x=\frac{1}{e^{\prime} e^{i}} \operatorname{Eam} \frac{2 K x}{\pi}, \\
& V_{2}=\frac{1}{e^{14}} \cdot \frac{2 K}{\pi} \cdot \int_{0}^{x} \triangle^{4} \mathrm{am} \frac{2 K x}{\pi} \partial x \\
& =\frac{e e}{3 e^{14}} \sin \xi \cdot \cos \xi \cdot \Delta \xi+\frac{2(2-e e)}{3 \cdot e^{14}} \mathrm{E} \operatorname{am} \frac{2 K x}{\pi}-\frac{1}{3 \cdot e^{t} e^{t}} \cdot \frac{2 K x}{\pi} .
\end{aligned}
$$

Horum $V, V^{2}, V^{2}$, unumquodque ipso

$$
\partial u=-\partial \cdot \operatorname{coam} \frac{2 K x}{x}=e^{\prime} \frac{\partial \xi}{\Delta^{x} \xi}
$$

*) Vid. Crelle Journal für die reine und angew. Mlathem. Tom. FV. pag. 373. 
multiplicandum et postea integrandum est, qua occasione duo integralia obviam fiunt, quae separatim tractentur oportet. Haec sunt:

$$
\frac{2 K}{\pi} \int_{0}^{x} x . \partial \cdot \operatorname{coam} \frac{2 K x}{\pi} ; \int_{0}^{x} \operatorname{Eam} \frac{2 K x}{\pi} \partial \cdot \operatorname{coam} \frac{2 K x}{\pi}
$$

$$
\text { §. } 6 .
$$

In formula priori, integratione per partes adhibita, eruitur

$$
\frac{2 K}{\pi} \int_{0}^{x} \partial \cdot \operatorname{coam} \frac{2 K x}{\pi}=\frac{2 K \pi}{\pi} \cdot \operatorname{coam} \frac{2 K x}{\pi}-\frac{2 K}{\pi} \int_{0}^{x} \operatorname{coam} \frac{2 K x}{\pi} \partial x
$$

In expressione vero pro amplitudine exstante *):

$$
\operatorname{am} \frac{2 K \pi}{x}=x+\frac{2 q \sin 2 x}{1+q^{2}}+\frac{2 q^{2} \sin 4 x}{2\left(1+q^{4}\right)}+\frac{2 q^{2} \sin 6 x}{3\left(1+q^{\circ}\right)}+\ldots
$$

$\frac{\pi}{2}-x$ loco $x$ ponamus, unde accipiemus aequationem:

$$
\operatorname{coam} \frac{2 K x}{\pi}=\frac{\pi}{2}-x+\frac{2 q \sin 2 x}{1+q^{2}}-\frac{2 q^{2} \sin 4 x}{2\left(1+q^{4}\right)}-\frac{2 q^{3} \sin 6 x}{3\left(1+q^{6}\right)}-\ldots
$$

- qua ipso $\frac{2 \pi}{\pi} \cdot \partial x$ multiplicata et postea integrata provenit:

$$
\begin{gathered}
\frac{2 K}{\pi} \int_{0}^{x} \cos \frac{2 K x}{\pi} \partial x \\
=\frac{2 K}{\pi}\left[\frac{1}{2} \pi x-\frac{3}{2} x x-\frac{q \cos 2 x}{1+q^{2}}+\frac{q^{2} \cos 4 x}{4\left(1+q^{4}\right)}-\frac{q^{2} \cos 6 x}{9\left(1+q^{\circ}\right)}+\ldots .\right] \\
+\frac{2 K}{\pi}\left[\frac{q}{1+q^{2}}-\frac{\pi}{4} \frac{q^{2}}{1+q^{4}}+\frac{q^{2}}{1+q^{6}}-\ldots\right] \\
=\frac{K x}{\pi}[\pi-x]+\frac{4 K}{\pi}\left[\frac{q \sin x^{2}}{1+q^{2}}-\frac{q^{2} \sin 2 x}{4\left(1+q^{4}\right)}+\frac{q^{2} \sin 3 x^{2}}{9\left(1+q^{6}\right)}-\ldots\right] \\
=\frac{K x}{\pi}[\pi-x]-\frac{4 K}{\pi} \sum \frac{(-q)^{n} \sin n x^{2}}{n \cdot m\left(1+q^{2 n}\right)},
\end{gathered}
$$

ubi numero $n$ tribuantur valores: $1,2,3, \ldots \ldots \infty$, itaque:

$$
\stackrel{2}{2} \frac{2 K}{\pi} \int_{0}^{x} x \cdot \partial \cdot \operatorname{coam} \frac{2 K x}{\pi}=-\frac{K x}{\pi}[2 u-x]+\frac{4 K}{\pi} \sum \frac{(-q)^{n} \sin n x^{2}}{n n \cdot\left(1+q^{2 n}\right)} \text {. }
$$

\section{\$. 7.}

Secundum integrale, quod nobis occurrit, est:

Cum vero sit :

$$
\int_{0}^{x} \operatorname{Eam} \frac{2 K x}{\pi} \cdot \partial \cdot \operatorname{coam} \frac{2 K x}{\pi} .
$$

$\mathbf{E}$ am $\frac{2 K x}{\pi}=\frac{2 K}{\pi} \int_{0}^{x} \Delta^{2} \operatorname{am} \frac{2 K x}{\pi} \partial x=\frac{2 K x}{\pi}-\frac{2 K}{\pi} e e \int_{0}^{x} \sin ^{2} \operatorname{am} \frac{2 K x}{\pi} \partial x$ erit e notatione Cl. Jacobi:

*) Vid. Jacobi Fundamenta nova theor. funct. ellipt. 9. 39. Nro. 24. pag. 102. 
2. Sohncke, motus corporum coelestium in medio resistente.

igitur:

$$
\frac{2 K}{\pi} \mathrm{E} \text { am } \frac{2 K x}{\pi}=\frac{2 K}{\pi}\left[\frac{2 \mathrm{E}^{\prime} x}{\pi}+\mathrm{Z}\left(\frac{2 \pi x}{\pi}\right)\right]
$$

$$
\begin{gathered}
\frac{2 K}{\pi} \int_{0}^{x} \operatorname{Eam} \frac{2 K x}{\pi} \cdot \partial \cdot \operatorname{coam} \frac{2 K x}{\pi} \\
=\frac{2 K}{\pi} \cdot \frac{2 E^{\prime}}{\pi} \int_{0}^{x} x \cdot \partial \cdot \operatorname{coam} \frac{2 K x}{\pi}+\frac{2 K}{\pi} \int_{0}^{x} Z\left(\frac{2 K x}{\pi}\right) \cdot \partial \cdot \operatorname{coam} \frac{2 K x}{\pi} .
\end{gathered}
$$

Priorem partem hujus integralis in sectione sexta illustravimus, qua re tantum altera pars restat enucleando. Hunc in finem seriem pro coamplitudine antea datam ratione $x$ differentiemus, deinde in seriem pro $\frac{2 K}{\pi} \mathrm{Z}\left(\frac{2 K x}{\pi}\right) *$ ducamus, denique integremus.

Formula vero pro coamplitudine:

$$
\operatorname{coam} \frac{2 K x}{\pi}=\frac{\pi}{2}-x+\frac{2 q \sin 2 x}{1+q^{2}}-\frac{2 q^{2} \sin 4 x}{2\left(1+q^{4}\right)}+\frac{2 q^{3} \sin 6 x}{3\left(1+q^{6}\right)}-\ldots
$$

secundum $x$ differentiata praebet:

$$
\begin{aligned}
& \text { d. } \operatorname{coam} \frac{2 K x}{x}=-\left[1-\frac{4 q \cos 2 x}{1+q^{2}}+\frac{4 q^{2} \cos 4 x}{1+q^{4}}-\frac{4 q^{3} \cos 6 x}{1+q^{6}}+\ldots\right] . \partial x \\
& =-\left[1+4 \Sigma \frac{(-q)^{n} \cos 2 n x}{1+q^{2 n}}\right] \cdot \partial x \text {, } \\
& n=1,2,3, \ldots \infty
\end{aligned}
$$

hace est ducenda in:

$$
\begin{aligned}
\frac{2 K}{\pi} Z\left(\frac{2 K x}{\pi}\right)= & 4 \cdot\left[\frac{q \sin 2 x}{1-q^{2}}+\frac{q^{2} \sin 4 x}{1-q^{4}}+\frac{q^{3} \sin 6 x}{1-q^{6}}+\ldots . . .\right] \\
= & 4 . \sum \frac{q^{n} \sin 2 n x}{1-q^{2 n}}, \\
& =12.3, \ldots x
\end{aligned}
$$

qua si multiplieatione revera utimur, accita formula trigononetrica:

$$
2 \sin \alpha \cos \beta=\sin (\alpha+\beta)+\sin (\alpha-\beta) \text {. }
$$

factorem ipsius $(-4 \sin 4 n x)$ adipiscimur $=$

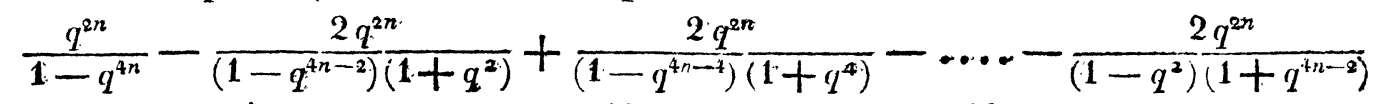

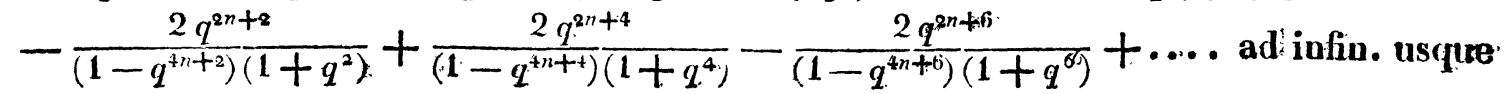

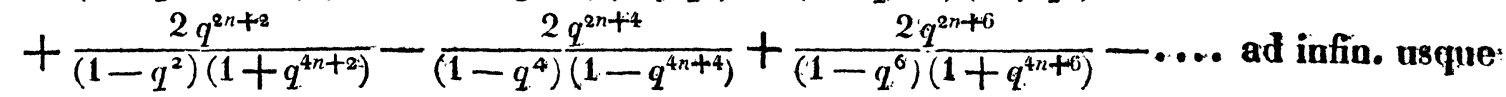
$\Sigma(-1)^{m} \frac{2 q^{2 n}}{\left(1-q^{4 n-2 m}\right)\left(1+q^{2 m}\right)}+\Sigma(-1)^{p} \frac{2 q^{2 n+8 p}}{\left(1-q^{4 n+2 p}\right)\left(1+q^{2 p}\right)}-\Sigma(-1)^{p} \frac{2 q^{2 n+8 p}}{\left(1-q^{2 p}\right)}\left(1+q^{4 n+2 p}\right) \cdot$ $m=0,1,2,3, \ldots(2 n-1)$

$$
p=1,2,3, \ldots \ldots
$$$$
p=1,2,3, \ldots \ldots \infty
$$

*) 1. c. §. 47 . pag. 133 ; 
Quia tamen omnes fractiones hic obviae in fractiones partiales discerpi possunt per formulas:

$$
\begin{aligned}
& \frac{2 q^{2 n}}{\left(1-q^{4 n-2 m}\right)\left(1+q^{2 m}\right)}=\frac{2 q^{2 n}}{1+q^{4 n}} \cdot\left[1+\frac{q^{4 n-2 m}}{1-q^{4 n-2 m}}-\frac{q^{2 m}}{1+q^{2 n}}\right], \\
& \frac{2 q^{2 n+2 p}}{\left(1-q^{4 n+2 p}\right)\left(1+q^{2 p}\right)}=\frac{2 q^{2 n}}{1+q^{4 n}} \cdot\left[\frac{q^{4 n+2 p}}{1-q^{4 n+2 p}}+\frac{q^{2 p}}{1+q^{2 p}}\right], \\
& \frac{2 q^{2 n+2 p}}{\left(1-q^{2 p}\right)\left(1+q^{4 n+2 p}\right)}=\frac{2 q^{2 n}}{1+q^{4 n}} \cdot\left[\frac{q^{2 p}}{1-q^{2 p}}+\frac{q^{4 n+2 p}}{1+q^{4 n+2 p}}\right]
\end{aligned}
$$

obtinemus coefficientem termini, qui $(-4 \sin 4 n x)$ continet:

$$
\begin{aligned}
& \quad=\frac{2 q^{2 n}}{1+q^{4 n}} \cdot\left[\Sigma(-1)^{m}+\Sigma(-1)^{m} \cdot \frac{q^{4 n-2 m}}{1-q^{4 n-2 m}}-\Sigma(-1)^{m} \cdot \frac{q^{2 m}}{1+q^{2 m}}\right] \\
& +\Sigma(-1)^{p} \frac{q^{4 n+2 p}}{1-q^{4 n+2 p}}+\Sigma(-1)^{p} \frac{q^{2 p}}{1+q^{2 p}}-\Sigma(-1)^{p} \frac{q^{2 p}}{1-q^{2 p}}-\Sigma(-1)^{p} \frac{q^{4 n+2 p}}{1+q^{4 n+2 p}}
\end{aligned}
$$

siquidem in summis brevitatis causa signo $\Sigma$ praefixo notatis, literae $i$ omnes valores $0,1,2,3, \ldots(2 n-1)$, literae $p$ vero omnes valores ab unitate ad infinitum usque tribuuntur.

Cum vero sit:

$$
\begin{gathered}
\sum(-1)^{m}=0 \\
m=0,1,2, \ldots(2 n-1) \\
\Sigma(-1)^{m} \frac{q^{4 n-2 m}}{1-q^{4 n-3 m}}+\Sigma(-1)^{p} \frac{q^{4 n+2 p}}{1-q^{4 n+2 p}}-\Sigma(-1)^{p} \frac{q^{2 p}}{1-q^{2 p}}=0
\end{gathered}
$$

$m=0,1,2, \ldots(2 n-1)$

$p=1,2,3, \ldots \infty$

$-\Sigma(-1)^{m} \frac{q^{2 m}}{1+q^{2 m}}-\Sigma(--1)^{p} \frac{q^{4 n+2 p}}{1+q^{4 n+2 p}}+\Sigma(-1)^{p} \frac{q^{2 p}}{1+q^{2 p}}=-\frac{1}{2}+\frac{q^{4 n}}{1+q^{4 n}}$, $m=0,1,2, \ldots(2 n-1)$

$$
p=1,2,3, \ldots . \infty
$$

nanciscimur :

unde terminus quaesitus fit:

$$
\frac{2 q^{2 n}}{1+q^{4 n}}\left[-\frac{1}{2}+\frac{q^{4 n}}{1+q^{4 n}}\right] \text { sive: }-\frac{q^{2 n}\left(1+q^{4 n}\right)}{\left(1+q^{4 n}\right)^{2}}
$$

$$
=\frac{4 q^{2 n}\left(1-q^{4 n}\right)}{\left(1+q^{4 n}\right)^{2}} \sin 4 n x .
$$

Eodem modo subsequens terminus eruitur:

$$
=-\frac{4 q^{2 n+1}\left(1-q^{4 n+2}\right)}{\left(1+q^{4 n}+2\right)^{2}} \sin (4 n+2) x,
$$

quare totum productum aequale fit:

$$
=4 \Sigma \frac{(-q)^{n}\left(1-q^{2 n}\right)}{\left(1+q^{2 n}\right)^{2}} \sin 2 n x .
$$

Hoc per $\partial x$ multiplicatum atque deinde inter limites 0 et $x$ integratum abit in: 
2. Sohncke, motus corporum coelestium in medio resistente.

$$
\begin{aligned}
& -2 \sum \frac{(-q)^{n}\left(1-q^{2 n}\right)}{n\left(1+q^{2 n}\right)^{2}} \cos 2 n x+2 \sum \frac{(-q)^{n}\left(1-q^{2 n}\right)}{n\left(1+q^{2 n}\right)^{2}} \\
& =4 \sum \frac{(-q)^{n}\left(1-q^{2 n}\right)}{n\left(1+q^{2 n}\right)^{2}} \sin n x^{2} . \\
& \quad n=1,2,3, \ldots \ldots \infty
\end{aligned}
$$

His collectis habebimus:

$$
\text { M. } \begin{aligned}
\int_{0}^{x} \operatorname{Eam} \frac{2 K x}{\pi} \cdot \partial \cdot \operatorname{coam} \frac{2 K x}{\pi}= & -\frac{\mathrm{E}^{\prime} x}{\pi} \cdot[2 u-x]+\frac{4 \mathrm{E}^{\prime}}{\pi} \Sigma \frac{(-q)^{n} \sin n x^{2}}{n n\left(1+q^{2 n}\right)} \\
& +\frac{2 \pi}{K} \Sigma \frac{(-q)^{n}\left(1-q^{2 n}\right) \sin n x^{2}}{n\left(1+q^{2 n}\right)^{2}},
\end{aligned}
$$

ubi pro $n$ omnes deinceps numeri integri ab unitate ad infinitum usque ponendi suut.

Idem integrale:

$$
\text { §. } 8 .
$$

$$
\int_{0}^{x} \operatorname{Eam} \frac{2 \pi x}{\pi} \cdot \partial \operatorname{coam} \frac{2 \pi x}{\pi},
$$

elegantiori methodo assequi possumus. Est enim in $\$ .5$. positum:

$$
\int_{0}^{w} \frac{\partial w}{\sqrt{\left(\mathrm{r}-e e \sin w^{2}\right)}}=K-\frac{2 K x}{\pi} \text { et } \int_{0}^{\frac{\pi}{2}} \frac{\partial \operatorname{ain} \frac{2 K x}{\pi}}{\sqrt{\left(1-e e \sin ^{2} \operatorname{am} \frac{2 K x}{\pi}\right)}}=K \text {, }
$$

e quibus ratione $q$ differentiatis (ubi $x$ tanquam constans, $e, K$ et $w$ vero tanquam variabiles considerari debent) profluunt:

$$
\begin{aligned}
& \text { a. } e \frac{\partial e}{\partial q} \cdot \int_{\cdot}^{w} \frac{\sin ^{2} w \partial w}{\left[1-e e \sin ^{2} w\right]^{\frac{3}{2}}}+\frac{\partial w}{\partial q} \cdot \frac{1}{r\left(1-e e \sin ^{2} w\right)}=\left[1-\frac{2 x}{\pi}\right] \cdot \frac{\partial K}{\partial q}, \\
& \text { b. } \frac{\partial K}{\partial q}=\left[\frac{1}{1-e c} \mathbf{E}^{\prime}-K\right] \frac{1}{e} \cdot \frac{\partial e}{\partial q} .
\end{aligned}
$$

Ut valorem ipsius $\frac{\partial e}{\partial q}$ obtineamus, advocemus formulam*):

Est vero $\left.{ }^{*}\right): q=e^{-\frac{\pi K^{\prime}}{K}}$ quod differentiatum exhibet

$$
\begin{aligned}
& \partial \frac{K^{\prime}}{K}=-\frac{\frac{s}{2} \pi \cdot \partial e}{c(1-e c) K} \\
& \text { quod differentiatum } \\
& \quad \partial \cdot \frac{K^{\prime}}{K}=-\frac{\partial q}{\pi q} .
\end{aligned}
$$

His comparatis impetramus:

$$
\frac{\partial e}{\partial q}=\frac{e(1-e e)}{2, q} \cdot\left(\frac{2 K}{\pi}\right)^{2}
$$

*) 1. c. \$. 32. pag 74. posito $a=b^{\prime}=1, a^{\prime}=b=0$ in formula:

*) 1. c. pag. 85 .

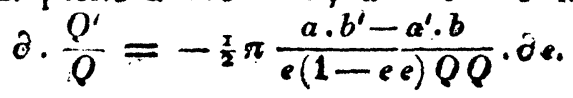

Crelle's Journal d. M. Bd.X. Ift. 1. 
quo valore in formula (b.) posito nanciscimur:

$$
\text { c. } \frac{\partial K}{\partial q}=\frac{1-e e}{2 q}\left(\frac{2 K}{\pi}\right)^{2}\left[\frac{1}{1-e e} \mathbf{E}^{\prime}-K\right]
$$

unde denique aequatio $(\alpha$.$) transit in sequentem:$

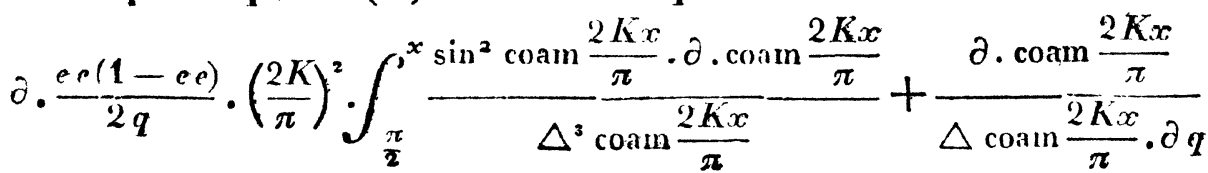

$$
\begin{aligned}
& =\frac{1}{2 q}\left(\frac{2 K}{\pi}\right)^{2}\left[\mathrm{E}^{\prime}-(1-e c) K\right] \cdot\left[1-\frac{2 x}{\pi}\right] \text {, }
\end{aligned}
$$

sive, cum sit :

$$
\begin{gathered}
e \varepsilon(1-e e) \int_{\frac{\pi}{2}}^{\dot{x}^{x} \sin ^{2} \operatorname{coan} \frac{2 K x}{\pi} \cdot \partial \cdot \operatorname{coam} \frac{2 K x}{\pi}}=-e e \int_{\frac{\pi}{2}}^{x} \cos ^{2} \operatorname{am} \frac{2 K x}{\pi} \cdot \partial \cdot \frac{2 K x}{\pi} \\
=(1-e e) \frac{2 K}{\pi}\left(x-\frac{\pi}{2}\right)-\operatorname{Eam} \frac{2 K x}{\pi}+\mathrm{E}^{\prime}
\end{gathered}
$$

in hanc:

$$
\begin{gathered}
-\frac{2 K}{\pi} \cdot \mathrm{E} \text { am } \frac{2 K x}{\pi} \Delta \operatorname{coam} \frac{2 K x}{\pi} \\
=-\frac{2 K}{\pi} \cdot \frac{2 E^{\prime}}{\pi} \cdot x \Delta \operatorname{coam} \frac{2 K x}{\pi}-\frac{q \pi}{K} \cdot \frac{\partial \cdot \operatorname{coam} \frac{2 K x}{\pi}}{\partial q},
\end{gathered}
$$

quae ipso $\partial x$ multiplicata, deinde inter limites 0 et $x$ integrata, hanc formam induit :

$$
\begin{gathered}
e \cdot \int_{0}^{x} \mathrm{E} \operatorname{am} \frac{2 K x}{\pi} \cdot \partial \cdot \operatorname{coam} \frac{2 K x}{\pi} \\
=\frac{2 \mathrm{E}^{k}}{\pi} \int_{0}^{x} x \cdot \partial \cdot \operatorname{coam} \frac{2 K x}{\pi}-\frac{q \pi}{K} \int_{0}^{x} \frac{\partial \cdot \operatorname{coam} \frac{2 K x}{\pi}}{\partial q} \cdot \partial x,
\end{gathered}
$$

quorum alterum terminum in $\$ .6$. computatione consecuti sumus:

$$
\begin{aligned}
& \text { ․ } \int_{0}^{x} x \cdot \partial \cdot \operatorname{coam} \frac{2 K x}{\pi}=-\frac{x}{2}[2 u-x]+2 \Sigma \frac{(-q)^{n} \sin ^{2} n x}{n n\left(1+q^{2 n}\right)} \text {, } \\
& n=1,2,3, \ldots \infty
\end{aligned}
$$

altèrum vero inveniemus, si expressionem pro coamplitudine propositam

$$
\begin{aligned}
\operatorname{coam} \frac{2 K_{x} x}{\pi} & =\frac{\pi}{2}-x+\frac{2 q \sin 2 x}{1+q^{2}}-\frac{2 q^{2} \sin 4 x}{2\left(1+q^{4}\right)}+\frac{2 q^{3} \sin 6 x}{3\left(1+q^{5}\right)}-\ldots \\
& =\frac{1}{2}-x-2 \sum \frac{(1-q)^{n} \cdot \sin 2 n x}{n\left(1+q^{2 n}\right)} \\
n=1,2,3, \ldots \infty &
\end{aligned}
$$

ratione $q$ differentiaverimus, quo pacto fit:

$$
\frac{\partial \cdot \operatorname{coam} \frac{2 K x}{\pi}}{\partial q}=2 \Sigma \frac{(-q)^{n-1}\left(1-q^{2 n}\right) \sin 2 n x}{\left(1+q^{2 n}\right)^{2}},
$$


quod ipso $\frac{\pi q}{K} \cdot \partial x$ multiplicatum et inter limites 0 et $x$ integratum, praebet:

f. $\frac{\pi q}{K} \int_{0}^{x} \frac{\partial . \operatorname{caom} \frac{2 K x}{\pi}}{\partial q} \cdot \partial x=-\frac{2 \pi}{K} \Sigma \frac{(-q)^{n}\left(1-q^{2 \eta}\right) \sin ^{2} n x}{n\left(1+q^{2 n}\right)^{2}}$.

Hinc illa aequatio $(e$.$) sic enucleata apparebit:$

$$
=-\int \frac{E^{\prime} x}{\pi}[2 u-x]+\frac{4 E^{\prime}}{\pi} \Sigma \frac{(-q)^{n} \sin ^{2} n x}{n n\left(1+q^{2 n}\right)}+\frac{2 \pi}{K} \Sigma \frac{(-q)^{n}\left(1-q^{2 n}\right) \sin ^{2} n x}{n\left(1+q^{2 n}\right)^{2}},
$$

quae eadem est atque aequatio (M.) in $\$ .7$.

In sectione quarta fuit:

$$
\text { §. } 9 .
$$

$$
\int_{0}^{u} R \cdot \partial u=4 e \int_{0}^{u} U \cdot \partial u+\int_{0}^{u} V \cdot \partial u-8 \int_{0}^{u} V_{2} \cdot \partial u+8 \int_{0}^{u} V_{2} \cdot \partial u \text {. }
$$

Si valores pro $U, V, V_{2}, V_{2}$, ibidem et in sectionibus sequentibus accuratius constitutos hic substituimus, facillima reductione adhibita sequitur:

$$
\begin{aligned}
& \int_{.}^{u t} R . \partial u=\frac{8}{3 e^{\prime 3}} \cdot\left[1+2 e \sin ^{2} \cdot \frac{1}{2} \xi-\Delta \xi\right]+\frac{4(1+3 e e)}{3 e^{\prime 4}} \cdot \operatorname{arc} \cdot\left[\operatorname{tang}=\frac{2 e e^{\prime} \sin ^{2} \frac{1}{2} \xi}{e^{\prime} e^{\prime}+e e \cos \xi}\right] \\
& -\frac{(5+3 e e) e^{\prime} e^{\prime} \cdot K-8(1+e e) E^{\prime}}{3 e^{\prime 4} \cdot \pi} \cdot\left[2 x \cdot u-x x-4 \Sigma \frac{(-q)^{n} \cdot \sin ^{2} n x}{n n \cdot\left(1+q^{2 n}\right)}\right] \\
& -\frac{1(i+1+e e)}{3 e^{14}} \cdot \frac{\pi}{K} \cdot \Sigma \frac{(-q)^{n}\left(1-q^{2 i n}\right) \sin ^{2} n x}{n\left(1+q^{2 n}\right)^{2}} .
\end{aligned}
$$

Hinc cum esset:

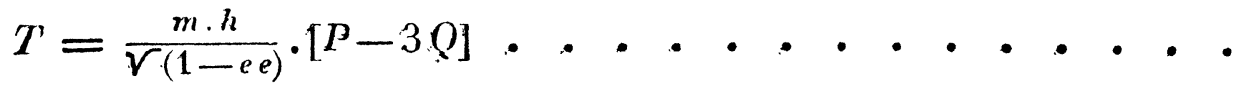

$$
\begin{aligned}
& P^{\prime}=\frac{2}{\lambda^{\prime} \sqrt{ } \lambda^{\prime}}\left[1-\lambda^{\prime}+\frac{4}{3} \frac{\lambda^{\prime}}{\left(1-\lambda^{\prime}\right)^{2}}\right]-\frac{2}{\lambda^{\prime} \sqrt{ } \lambda^{\prime}}\left[1+\frac{4}{3} \frac{\lambda^{\prime}}{\left(1-\lambda^{\prime}\right)^{3}}\right] \Delta^{3} \varphi+\frac{2}{\sqrt{ } \lambda^{\prime}} \Delta \varphi \\
& +2 \cdot \operatorname{arc}\left[\operatorname{tang}=\frac{V\left(\lambda^{\prime}(1-\Delta p)\right)}{\lambda^{\prime}+\Delta p}\right] . . .
\end{aligned}
$$

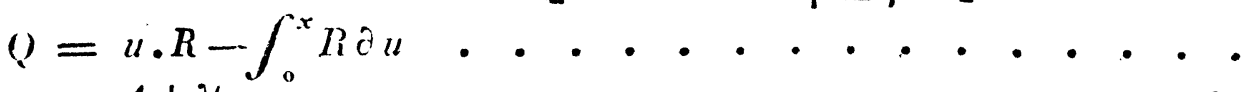

$$
\begin{aligned}
& R=\frac{1+\lambda^{\prime}}{3 \lambda^{\prime} \lambda^{\prime}} \cdot\left[\lambda \lambda \sin \varphi \cdot \cos \varphi \cdot \Delta \varphi+2\left(1+\lambda^{\prime} \lambda^{\prime}\right) \mathbf{E}(\varphi)-\lambda^{\prime} \lambda^{\prime} \mathbf{F}(\varphi)\right]^{\circ} .
\end{aligned}
$$

cradit :

$$
\begin{aligned}
i= & -m \cdot h \frac{\left(1+\lambda^{\prime}\right)}{\lambda^{\prime} \lambda^{\prime}} \cdot\left[1+\lambda^{\prime}+\lambda^{\prime} \lambda^{\prime}+\frac{\left(1-\frac{2}{8} \lambda^{\prime}+\lambda^{\prime} \lambda^{\prime}\right) \Delta^{2} \varphi}{\left(1-\lambda^{\prime}\right)^{3}}\right] \cdot \Delta \varphi \\
& +\frac{\left.m \cdot h \cdot{ }^{\prime} 1+\lambda^{\prime}\right)}{\lambda^{\prime} \lambda^{\prime}\left(1-\lambda^{\prime}\right)^{2}} \cdot\left[2-\frac{5}{3} \lambda^{\prime}+\lambda^{\prime} \lambda^{\prime}-\lambda^{\prime 3}+\lambda^{\prime 4}\right] \\
& +m \cdot h \frac{\left(1-\lambda^{\prime 5}\right)\left(1+\lambda^{\prime}\right)}{\lambda^{\prime} \lambda^{\prime} V \lambda^{\prime}\left(1-\lambda^{\prime}\right)} \operatorname{arc}\left[\operatorname{tang}=\frac{V \lambda^{\prime}(1-\Delta \varphi)}{\lambda^{\prime}+\Delta \varphi}\right] \\
& -\frac{m h \cdot u\left(1+\lambda^{\prime}\right)^{2}}{2 \lambda^{\prime} \lambda^{\prime} V \lambda^{\prime}} \cdot\left[\lambda \lambda \sin \varphi \cdot \cos \varphi \cdot \Delta \varphi+2\left(1+\lambda^{\prime} \lambda^{\prime}\right) \mathbf{E}(\varphi)-\lambda^{\prime} \lambda^{\prime} \mathbf{E}(\varphi)\right]
\end{aligned}
$$




$-\frac{m h \cdot\left[(5+3 e e) e^{\prime} e^{\prime} K-8(1+e e) E^{\prime}\right]}{e^{16} \pi}\left[2 x \cdot u-x x+\frac{4 q \cdot \sin ^{2} \cdot x}{1+q^{2}}-\frac{4 q^{2} \sin ^{2} \cdot 2 x}{4\left(1+q^{4}\right)}+\frac{4 q^{3} \sin ^{2} 3 x}{9\left(1+q^{6}\right)}-\ldots ..\right]$
$+\frac{16 m h \cdot(1+e e)}{e^{\prime 6}} \cdot \frac{\pi}{K} \cdot\left[\frac{q\left(1-q^{2}\right) \sin ^{2} x}{\left(1+q^{2}\right)^{2}}-\frac{q^{2}\left(1-q^{4}\right) \sin ^{2} 2 x}{2\left(1+q^{4}\right)^{2}}+\frac{q^{3}\left(1-q^{6}\right) \sin ^{2} 3 x}{3\left(1+q^{6}\right)^{2}}-\ldots.\right]$.

§. 10.

Hic valor ipsius $T$ calculando minus aptus est, quoties excentricitas $e$ unitati proxime accedit, pro valore enim $e=1$ fit $T=\frac{0}{0}$; verumtamen huic casui transformatio serierum favet.

Ponatur enim in formula nona 1. c. §. 39. pag. 99.

$\left.\log \sqrt{\left(1-\sin \operatorname{am} \frac{2 K x}{\pi}\right.}\right)=\log V\left(\frac{2+\sin x}{1-\sin x}\right)+\frac{4 q \sin x}{1-q}-\frac{4 q^{3} \sin 3 x}{3\left(1-q^{3}\right)}+\frac{4 q^{8} \sin 5 x}{5\left(1-q^{5}\right)}-\ldots$

ix loco $x$, quo facto, adhibita aequatione nota:

expressio:

$$
\sin \operatorname{am} i u=i \operatorname{tang} \operatorname{am}\left(u, k^{\prime}\right) \text {, }
$$

$$
\log \sqrt{\left(\frac{1+\sin \operatorname{am} \frac{2 K x}{\pi}}{1-\sin \operatorname{ann} \frac{2 K x}{\pi}}\right)}
$$

convertitur in:

$$
-i \operatorname{am}\left(\frac{2 K x}{\pi}, k^{\prime}\right) \text {. }
$$

Praeterea si facimus: $\sin i x=i \operatorname{tang} y$, quod idem valet ac

$$
e^{x}=\operatorname{tang}\left(\frac{1}{4} \pi+\frac{1}{2} y\right)
$$

ubi $e$ basin logarithmorum naturalium significat, prodit:

$$
\log V\left(\frac{1+\sin x}{1-\sin x}\right)=-i y,
$$

unde formula citata transformatur in:

$$
\operatorname{am}\left(\frac{2 K x}{\pi}, k^{\prime}\right)=
$$

2. $\operatorname{arc}\left(\operatorname{tang}=e^{x}\right)-\frac{1}{2} \pi-\frac{2 q\left(e^{x}-e^{-x}\right)}{1-q}+\frac{2 q^{3}\left(e^{3 x}-e^{-3 x}\right)}{3\left(1-q^{3}\right)}-\frac{2 q^{8}\left(e^{3 x}-e^{-3 x}\right)}{5\left(1-q^{6}\right)}+\ldots$

Si vero $k$ pro $k^{\prime}$ scribimus, quo

abeunt, eruimus :

$$
\begin{gathered}
K \text { in } K^{\prime}, \\
K^{\prime} \text { in } K, \\
q=e^{-\frac{\pi K^{\prime}}{K}} \text { in } q^{\prime}=e^{-\frac{\pi \pi}{K^{\prime}}}
\end{gathered}
$$

$\operatorname{am}\left(\frac{2 K^{\prime} x}{\pi}, k\right)=2 \operatorname{arc}\left(\operatorname{tang}=e^{x}\right)-\frac{1}{2} \pi-\frac{2 q^{\prime}\left(e^{x}-e^{-x}\right)}{1-q^{\prime}}+\frac{2 q^{\prime}\left(e^{3 x}-e^{-3 x}\right)}{3\left(1-q^{\prime 3}\right)}-\ldots$ 
Posito etiam $\frac{K x}{K^{\prime}}$ pro $x$, fit terminus generalis:

$$
=\frac{2(-1)^{n} q^{\prime^{n}} \cdot\left[e^{\frac{n K x}{K^{\prime}}}-e^{-\frac{n K x}{K^{\prime}}}\right]}{n\left(1-q^{\prime^{\prime \prime}}\right)},
$$

sive pro $q^{\prime}$ valore ejus $=e^{-\frac{\pi K}{K^{\prime}}}$ scripto:

itaque:

$$
\begin{aligned}
& =\frac{2(-1)^{n} \cdot e^{\frac{n K \pi}{K^{\prime}}} \cdot\left[e^{\frac{n K x}{K^{\prime}}}-e^{-\frac{n K x}{K^{\prime}}}\right]}{n \cdot\left[1-e^{-\frac{n K \pi}{K^{\prime}}}\right]} \\
& =\frac{2(-1)^{n} \cdot\left[e^{\frac{n K x}{K^{\prime}}}-e^{\left.-\frac{n K x}{K^{\prime}}\right]}\right.}{n \cdot\left[e^{\frac{n K \pi}{K^{\prime}}}-1\right]} ;
\end{aligned}
$$

$$
\text { g. } \begin{aligned}
\operatorname{am} \frac{2 K x}{\pi}= & 2 \cdot \operatorname{arc}\left(\operatorname{tang}=e^{\frac{K x}{K^{\prime}}}\right)-\frac{1}{2} \pi-\frac{2 \cdot\left[e^{\frac{K x}{K_{1}}}-e^{-\frac{\hbar x}{K^{\prime}}}\right]}{e^{\frac{K_{\pi}}{K^{\prime}}}-1} \\
& +\frac{2 \cdot\left[e^{\frac{3 K x}{K^{\prime}}}-e^{-\frac{3 K x}{K^{\prime}}}\right]}{3 \cdot\left[e^{\frac{3 K \pi}{K^{\prime}}}-1\right]}-\ldots,
\end{aligned}
$$

quam seriem vel maxime convergentem esse, si excentricitas unitati appropinquat, neminem fugit.

Hujus formulae ope series illas in expressione ipsius $T$ exstantes, quae magna excentricitate posita non satis convergunt, calculando aptiores facere possumus;

altera enim est:

altera vero:

$$
=\int_{0}^{x} \operatorname{coam} \frac{2 K x}{\pi} \cdot \partial x
$$

$$
=\int_{0}^{x} \frac{\partial \cdot \operatorname{coam} \frac{2 K x}{\pi}}{\partial q} \cdot \partial x
$$

Si igitur aequationem ( $g^{\circ}$ ) per $\partial x$ multiplicamus atque deinde inter limites 0 et $x$ integramus, postquam $\frac{\pi}{2}-x$ loco $x$ positum est, nanciscimur valorem seriei (․) $\mathfrak{L}_{\text {. }}$ 6.; atque si eandem aequationem ratione $q$ differentiamus, postquam $\frac{\pi}{2}-x$ loco $x$ positum est, deinde per $\partial x$ multiplicamus, denique inter limites 0 et $x$ integramus, nanciscimur valorem seriei $(f)$ \$. 8.

\section{§. 11.}

$U$ t in universum valorem $T$ magna excentricitate posita in seriem secundum potestates ipsius $(1-e)$ evolvamus, expressionem \$. 1. F. IV. 
rursus accipiamus: ibi fuit:

Ponamus :

$$
\begin{aligned}
\partial T & =\frac{m V p}{V(1-e e)} \cdot\left[\frac{2(1+e e)}{e}+e(1+e \cdot \cos u)\right] \frac{[1+e \cdot \cos u] \cdot \sin u \cdot \partial u}{[1-e \cdot \cos u]^{2} V\left(1-e e \cdot \cos ^{2} u\right)} \\
& -\frac{3 m V p}{V(1-e e)}\left[\frac{1+e \cdot \cos u}{1-e \cdot \cos u}\right]^{2} \frac{u \cdot \partial u}{V\left(1-e e \cos ^{2} u\right.} \cdot
\end{aligned}
$$

$$
\begin{gathered}
e=1-8 \\
\operatorname{tang} \frac{1}{2} u=V\left(\frac{1-e}{1+e}\right) \cdot \operatorname{tang} \frac{1}{2}(v-w)=V\left(\frac{\delta}{2-\delta}\right) \cdot \tau
\end{gathered}
$$

si tang $\frac{\pi}{2}(v-w)$ brevitatis causa signo redditur.

His positis invenimus:

$$
\begin{aligned}
& \cos u=\frac{1-\operatorname{tang}^{2} \frac{\pi}{2} u}{1+\operatorname{tang}^{2} \frac{1}{2} u}=\frac{1-\frac{1}{2} \delta(1+\tau \tau)}{1-\frac{1}{2} \delta(1-\tau \tau)}, \\
& \sin u=\frac{2 \operatorname{tang} \frac{1}{2} u}{1+\operatorname{tang}^{2} \frac{1}{2} u}=\frac{V(\delta(2-\delta)) \cdot \tau}{1-\frac{3}{2} \delta(1-\tau \tau)}, \\
& 1+e \cdot \cos u=\frac{2 \cdot\left[\left(1-\frac{\pi}{2} \delta\right)^{2}+\frac{\pi}{4} \delta^{2} x \tau\right]}{1-\frac{3}{2} \delta(1-\tau \tau)} \text {, } \\
& 1-e, \cos u=\frac{\delta\left(1-\frac{\pi}{2} \delta\right)(1+x \tau)}{1-\frac{1}{2} \delta(1-x \tau)} \\
& r\left(1-e e \cdot \cos ^{2} u\right)=\frac{V(\delta(2-\delta)) \cdot V(1+\tau \pi) \cdot V\left(\left(1-\frac{1}{2} \delta\right)^{3}+\frac{x}{4} \delta^{2} x \tau\right)}{1-\frac{1}{2} \delta(1-\tau \tau)}, \\
& \partial u=\frac{\sqrt{ }(\delta(2-\delta)) \cdot \hat{c} \tau}{1-\frac{1}{2} \delta(1-\tau)}, \\
& u=2 \cdot \operatorname{tang} \frac{\mathrm{T}}{2} u\left[1-\frac{\mathrm{T}}{3} \operatorname{tang}^{2} \frac{\mathrm{T}}{2} u+\frac{\mathrm{T}}{5} \operatorname{tang}^{4} \frac{\mathrm{T}}{2} u-\frac{1}{7} \operatorname{tang}^{5} \frac{\mathrm{r}}{2} u+\ldots\right] \\
& =\frac{2 V(\delta(2-\delta))}{2-\delta} \cdot \tau \cdot\left[1-\frac{1}{3}\left(\frac{\delta}{2-\delta}\right) \cdot \tau^{2}+\frac{1}{3}\left(\frac{\delta}{2-\delta}\right)^{2} \tau^{4}-\frac{1}{7}\left(\frac{\delta}{2-\delta}\right)^{3} \tau^{6}+\ldots .\right] \cdot
\end{aligned}
$$

Itaque habes:

$$
\begin{gathered}
\partial T=\frac{2 m h \cdot V\left[\left(1-\frac{1}{2} \delta\right)^{2}+\frac{\pi}{4} \delta^{2} \tau \tau\right] \cdot \tau \cdot \partial \tau}{\delta \delta \cdot\left(1-\frac{1}{2} \delta\right)^{2}(1+\tau \tau)^{2} V(1+\tau \tau)} \cdot \frac{2}{1-\delta}+3(1-\delta)+(1-\delta)^{2} \frac{1-\frac{1}{2} \delta(1+\tau \tau)}{1-\frac{1}{2} \delta(1-\tau \tau)} \\
\left.-\frac{3\left(2-\frac{\delta}{2}\right)^{2}}{2-\delta}\left[1+\left(\frac{\delta}{2-\delta}\right)^{2} \tau \tau\right] \cdot\left[1-\frac{1}{3}\left(\frac{\delta}{2-\delta}\right) \tau^{2}+\frac{3}{5}\left(\frac{\delta}{2-\delta}\right)^{2} \tau^{4}-\ldots\right]\right\},
\end{gathered}
$$

sive terminis et qui $\delta$ omnino non continent et qui in primam potestatem ipsius $\delta$ ducti sunt, ejectis, quia sese destruunt, in parenthesi expressio restat, quae secunda potestate ipsius $\delta$ multiplicata est, unde oritur:

$\partial T=\frac{m \cdot h V\left[\left(1-\frac{1}{2} \delta\right)^{2}+\frac{\pi}{4} \delta^{2} \tau \tau\right] \cdot \tau}{\left(1-\frac{1}{2} \delta \delta^{2}(1+\tau \tau)^{2} \sqrt{(1+\tau \tau)}\right.} \cdot \frac{(3-\delta)(2-\delta)}{1-\delta}+\frac{1}{2} \tau \tau \frac{(3+\tau \tau-2 \delta)(2-\delta)}{1-\frac{1}{2} \delta(1-\tau \tau)}$

$$
\left.-3 \tau \tau \cdot\left[\left(1+\frac{1}{5} \tau \tau\right)-\left(\frac{\delta}{2-\delta}\right) \tau \tau\left(\frac{1}{3}+\frac{1}{7} \tau \tau\right)+\left(\frac{\delta}{2-\delta}\right)^{2} \tau^{4}\left(\frac{1}{5}+\frac{1}{9} \tau \tau\right) \ldots\right]\right\} \text {. }
$$

Ut harc omnia secundum potestates ipsius $\delta$ ordinemus, advocato notationis more, e quo per $\mathbf{P}_{m}^{n} n^{\text {tami }}$ crefficientem binomialem evolutionis $m^{\text {in }}$ potestatis signamus, esse scimus: 


$$
\begin{gathered}
\frac{(3-\delta)(2-\delta)}{1-\delta}=\Sigma \cdot\left[6 \cdot \mathbf{P}_{-1}^{n}+5 \cdot \mathbf{P}_{-1}^{n-1}+\mathbf{P}_{-i}^{n-\tau}\right] \cdot(-\delta)^{n}, \\
\frac{\Sigma \tau \tau(3+\tau \tau-2 \delta)(2-\delta)}{1-\frac{1}{2} \delta(1-\tau \tau)}=\Sigma \cdot\left[\tau \tau(3+\tau \tau) \mathbf{P}_{-1}^{n} \frac{(1-\tau \tau)^{n}}{2^{n}}+\tau \tau(7+\tau \tau) \mathbf{P}_{-1}^{n-1} \frac{(1-\tau \tau)^{n-1}}{2^{n}}\right. \\
\left.+\tau \tau \mathbf{P}_{-1}^{n-2} \frac{(1-\tau \tau)^{n-2}}{2^{n-2}}\right\} \cdot(-\delta)^{n},
\end{gathered}
$$

deinde terminus generalis seriei in $3 \tau \tau$ multiplicatae, hic est:

$$
\frac{\tau^{2 m}(-\delta)^{m}}{2^{m}\left(1-\frac{1}{2} \delta\right)^{m}} \cdot\left[\frac{1}{2 m+1}+\frac{1}{2 m+5} \tau \tau\right]
$$

rerum ipsius $\left(1-\frac{11}{2} \delta\right)^{m}$ terminus generalis hic:

mude tota series fit :

$$
\mathbf{P}_{-m}^{i} \cdot \frac{(-\delta)^{i}}{2^{i}}
$$

$$
=\sum \mathrm{P}_{-m}^{i} \frac{\tau^{2 m}}{2^{m+i}} \cdot\left[\frac{1}{2 m+1}+\frac{1}{2 m+5} \cdot \tau \tau\right](-\delta)^{m+i},
$$

sive ipso $n$ pro $m+i$ scripto:

$$
=\sum \mathbf{P}_{-m}^{n-m} \frac{\boldsymbol{\tau}^{2 / n}}{2^{n}}\left[\frac{1}{2 m+1}+\frac{1}{2 m+5} \tau \tau\right](-\delta)^{n} \text {. }
$$

Denique igitur habebimus:

$$
\begin{gathered}
\partial T=\frac{m h V\left[\left(1-\frac{1}{2} \delta\right)^{2}+\frac{\tau}{2} \delta^{2} \tau \tau\right] \cdot \tau \cdot \partial \tau}{\left(1-\frac{1}{2}\right)^{3}(1+\tau \tau)^{2} V(1+\tau \tau)} \cdot \Sigma \cdot\left\{b \cdot \mathbf{P}_{-1}^{n}+5 \mathbf{P}_{-1}^{n-1}+\mathbf{P}_{-1}^{n-\tau}\right. \\
+\tau \tau(3+\tau \tau) \mathbf{P}_{-1}^{n}\left(\frac{1-\tau \tau}{2}\right)^{n}+\frac{\tau}{2} \tau \tau(7+\tau \tau) \mathbf{P}_{-1}^{n-1}\left(\frac{1-\tau \tau}{2}\right)^{n-1}+\tau \tau \mathbf{P}_{-1}^{n-2}\left(\frac{1-\tau \tau}{2}\right)^{n-2} \\
\left.-\frac{3 \tau^{2 m+2}}{2^{n}}\left(\frac{1}{2 m+1}+\frac{1}{2 m+5} \tau \tau\right) \mathbf{P}_{-m}^{n-m}\right\} \cdot(-\delta)^{n},
\end{gathered}
$$

ubi signum $\Sigma$ summam omnium terminorum innuit, qui omnibus valoribus $0,1,2,3 \ldots \infty$ pro $: 2$, et omuibus valoribus $0,1,2, \ldots n$ pro $m$ positis parantur.

Cum vero valoribus ipso 2 majoribus pro $n$ positis expressio multo magis contrahi possit, quia tum nulla coefficiens binomialis evanescit atque omnes ad potestatem -1 pertinentes $=+1$ aut $=-1$ funt, terminos non solum a $\delta$ non dependentes, sed etiam qui prima et secunda potestate ipsius $\delta$ sint multiplicati, a ceteris separabimus, quo facto impetratur : $\partial T=\frac{m h \cdot V\left[\left(1-\frac{\tau}{2} \delta\right)^{2}+\frac{\pi}{4} \delta^{2} \tau \tau\right] \cdot \tau \cdot \partial \tau}{\left(1-\frac{\pi}{2} \delta\right)^{3}(1+\tau \tau)^{2} V(1+\tau \tau)} \cdot\left\{2\left(3+\frac{\tau}{5} \tau^{4}\right)+\left(1-2 \tau \tau-\tau^{4}-\frac{2}{7} \tau^{6}\right) \delta\right.$

$$
\begin{gathered}
+\left(2+\frac{1}{2} \tau^{2}+\frac{1}{3} \sigma \tau^{n}+\frac{1}{\sigma} \tau^{n}\right) \delta^{2}+\Sigma\left[2+\tau^{4}\left(\frac{1-\tau \tau}{2}\right)^{n-2}\left(\frac{1+\tau \tau}{2}\right)^{2}\right](+\delta)^{n} \\
\left.-3 \Sigma \tau^{n}\left[\frac{1}{2 m-1-1}+\frac{1}{2 m+5} \tau \tau\right] \frac{t^{2 m+2}}{2^{n}} \cdot(-\delta)^{n}\right\},
\end{gathered}
$$

ubi in tepminis, gatsis signum $\Sigma$ praefixum est, ipsi $n$ omnes valores: $j, \frac{1}{x}, 5, \ldots \ldots$, ipsi $m$ omnes valores: $1,2,3, \ldots$, bibuen 1 i sunt. 
2. Solncke, motus corporum coelestium in medio resistente.

Si illi tantum termini considerantur et qui a $\delta$ non pendent et qui prima et secunda potestate ipsius $\delta$ sunt multiplicati, habemus:

$$
\begin{aligned}
& \partial T=m \cdot h \frac{\tau \cdot \partial \tau}{(1+\tau \tau)^{2} V(1+\tau \tau)} \cdot {\left[2\left(3+\frac{\tau}{5} \tau^{4}\right)+\left(\tau-2 \tau \tau-\frac{3}{5} \tau^{4}-\frac{2}{7} \tau^{6}\right) \delta\right.} \\
&+\left(\frac{15}{2}-\frac{5}{4} \tau \tau-\frac{1}{5} \tau^{4}+\frac{3}{14} \frac{\tau}{5} \tau^{6}+\frac{\tau}{6} \tau^{3}\right) \delta^{2},
\end{aligned}
$$

igitur integratione inter limites 0 et $\tau$ instituta:

$$
\begin{aligned}
T=m \cdot h \cdot & {\left[14-\frac{2\left(y-12 \tau \tau-3 \tau^{4}\right)}{(1+\tau \tau) V(1+\tau \tau)}+\frac{113}{105} \delta-\frac{\left(113-198 \tau \tau+3 \tau^{4}+10 \tau^{6}\right.}{105(1+\tau \tau) V(1+\tau \tau)} \delta\right.} \\
+ & \frac{617}{315} \delta^{2}-\frac{\left(2468-1023 \tau \tau+138 \tau^{4}+19 \tau^{\circ}-42 \tau^{4}\right)}{1260(1+\tau \tau) V(1+\tau \tau)} \delta^{2} .
\end{aligned}
$$

Regiom. 1. Novbr. 1832. 